(C) 2008 The Japan Society of Applied Physics

\title{
In(Ga)As Quantum Rings for Terahertz Detectors
}

\author{
Jong-Horng DAI, Jheng-Han LEE, Yi-Lung LIN, and Si-Chen LEE* \\ Department of Electrical Engineering and Graduate Institute of Electronics Engineering, National Taiwan University, Taipei 10617, Taiwan
}

(Received October 1, 2007; accepted November 28, 2007; published online April 25, 2008)

The $\operatorname{In}(\mathrm{Ga})$ As quantum ring infrared photodetector $(\mathrm{QRIP})$ were investigated. The quantum ring $(\mathrm{QR})$ is transformed from $500^{\circ} \mathrm{C}$ grown 2.2 ML quantum dot (QD) with QD annealing time $\sim 30 \mathrm{~s}$. The QRs show the broad PL spectrum which is blue shifted from QD about $100 \mathrm{meV}$. The resultant QRIP is a broadband terahertz detector. The response extends from 3 to $100 \mathrm{THz}$ with peak responsivity of $127 \mathrm{~mA} / \mathrm{W}$ at $23 \mu \mathrm{m}$ which has a spectral detectivity of $2.3 \times 10^{11} \mathrm{~cm} \mathrm{~Hz}^{1 / 2} / \mathrm{W}$ at $10 \mathrm{~K}$ under 1.2 V bias. [DOI: 10.1143/JJAP.47.2924]

KEYWORDS: terahertz detector, quantum dots, quantum rings, far-infrared

Terahertz $(\mathrm{THz})$ radiation covers a broad frequency range from $100 \mathrm{GHz}$ to $40 \mathrm{THz}$. It can be applied in the areas of imaging, communication, medical diagnosis, environmental control, health monitoring and biochemical identification. ${ }^{1-4)}$ There are few suitable detectors operating in this frequency range. Typical quantum well (QW) detectors using AlGaAs/ $\mathrm{GaAs}$ heterojunction structures with critical Al fractions are not suitable for normal-incidence and high-temperature operations. ${ }^{5-7)}$ Recently, the InAs/GaAs quantum dot infrared photodetector (QDIP) has demonstrated its operability in the far-infrared (FIR) region. ${ }^{8,9)}$ However, the cutoff frequency is limited to less than $40 \mu \mathrm{m}$. During the growth of an InAs/GaAs quantum dot (QD), it is difficult to control the InAs dot size to a small value uniformly; hence, it is difficult to achieve a small confined-to-conduction band transition. The growth of an InAs/GaAs quantum ring (QR) is similar to that of $\mathrm{QD} .{ }^{10-13)}$ The small confined $\mathrm{QR}$ structure is suitable for the requirement. A $2 \mathrm{~nm}$ GaAscapped layer was deposited, surrounding the InAs QDs, and the sample was annealed at a higher temperature to evaporate the central uncovered InAs dot, as shown in Fig. 1. Finally, the edge of the embedded InAs dot and the GaAs capped layer are mixed together to form an $\mathrm{In}(\mathrm{Ga}) \mathrm{As}$ ring structure, The confinement of $\mathrm{QR}$ is limited by the capped layer thickness. Therefore, $\operatorname{In}(\mathrm{Ga})$ As provides smaller confined-to-conduction band transitions that can be applied to detectors in the FIR range. This structure combines the advantages of QWs and QDs in terms of size controllability and normal incidence availability. In this work, we fabricated a broadband QR terahertz detector. The response was extended from 3 to $100 \mathrm{THz}$ which has a peak spectral responsivity of $127 \mathrm{~mA} / \mathrm{W}$ at $13 \mathrm{THz}$ under $1.2 \mathrm{~V}$ bias.

In $(\mathrm{Ga}) \mathrm{As} \mathrm{QRs}$ were grown by molecular beam epitaxy (MBE) using the Stranski-Krastanov growth mode. Selfassembled 2.2 ML InAs QDs were first grown on a GaAs substrate and covered by a $2 \mathrm{~nm}$ GaAs partial capping layer and then annealed for $30 \mathrm{~s}$ at $500^{\circ} \mathrm{C} .{ }^{14-16)}$ The original QDs had an average height of $8 \mathrm{~nm}$. The $2 \mathrm{~nm}$ GaAs layer was insufficient to cover entire dot only the hillside of the dot, hence the central dot was exposed to As2 vapor. This satisfies the conditions for transformation into QR. ${ }^{17)}$ The atomic force microscopy (AFM) image of the QR is shown in Fig. 2(a). It has a depth of $1.1 \mathrm{~nm}$ and an inner diameter of $10 \mathrm{~nm}$. The cross-sectional transmission electron microscopy

*E-mail address: sclee@cc.ee.ntu.edu.tw

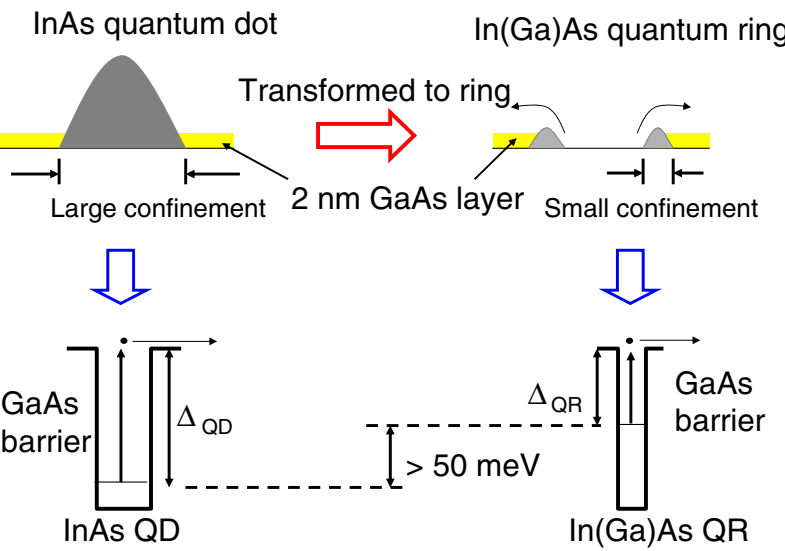

Fig. 1. (Color online) Schematic band structure of QR showing the lower energy transition as compared to that of a precursor QD.

(XTEM) shows the $\operatorname{In}(\mathrm{Ga}) \mathrm{As} \mathrm{QR}$ region [Fig. 2(b)]. It demonstrates that the ring region between the outer and inner diameters is narrower than normal QD that provides a smaller confined region than QD does. The center has exuded and the $\operatorname{In}(\mathrm{Ga})$ As region surround it remains small. It has been proved that the longer QD annealing time leads to larger strain relief in the higher aspect ratio QDs. ${ }^{18)}$ The same $2 \mathrm{~nm}$ thick GaAs capped layer on QDs results in different InAs buried regions. The small QD has the larger buried region and the high aspect ratio QD has the less. After out diffusion of InAs from central part of QD, the QR is formed. The $\operatorname{In}(\mathrm{Ga})$ As ring is smaller in size when QD has high aspect ratio. The higher aspect ratio dot results in a smaller confined ring. The PL spectra of QR and its precursor QD were measured. The PL peak wavelengths show a large blue-shifted from QD to QR, hence, the bandgap of $\mathrm{QR}$ is larger and the band discontinuity $\Delta_{\mathrm{QR}}$ is smaller as shown in Fig. 1. Our device is applied to the quantum structure to replace the intrinsic layer of the typical QDIP $\mathrm{n}-\mathrm{i}-\mathrm{n}$ structure. The ten periods of QR are embedded in the $50 \mathrm{~nm}$ undoped GaAs barrier within the $500 \mathrm{~nm}$ top and $1 \mu \mathrm{m}$ bottom contact layers sandwiching it. The schematic of the detector structure is shown in Fig. 2(c).

The detector with an area of $150 \times 150 \mu \mathrm{m}^{2}$ is fabricated after the above two-step process. The device is measured under an edge coupling scheme with a cryostat. The temperature-dependent dark and ambient background irradiation current-voltage characteristics are measured using by HP $4156 \mathrm{~B}$ precision semiconductor parameter analyzer. At a lower temperature of $20 \mathrm{~K}$, dark current is restrained 


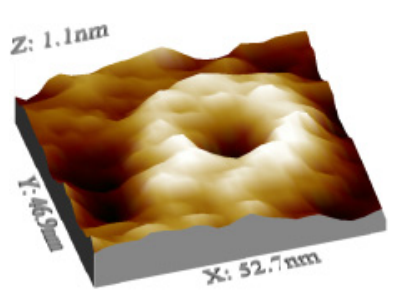

(a)

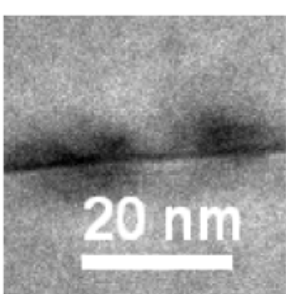

(b)

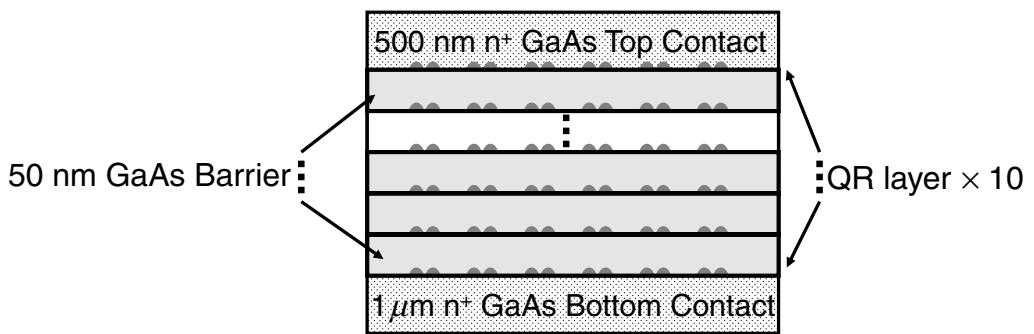

(c)

Fig. 2. (Color online) (a) Three-dimensional AFM image of a single QR. (b) XTEM image of QR. (c) Schematic device structure with self-organized In(Ga)As quantum rings.

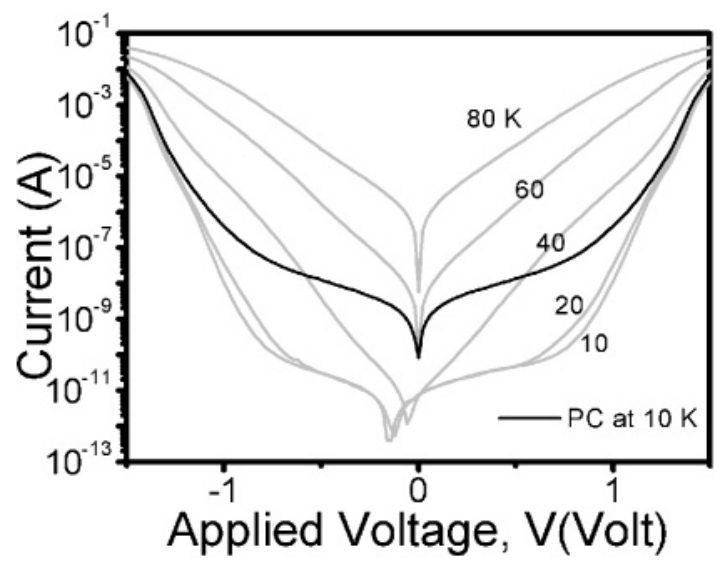

Fig. 3. Dark current as functions of applied voltage of the detector from 10 to $80 \mathrm{~K}$. The bold curve represents photocurrents measured at $10 \mathrm{~K}$ when the device is illuminated from ambient background radiation through the cryostat window.

under $2.3 \times 10^{-10} \mathrm{~A}$. The BLIP temperature of the detector is $40 \mathrm{~K}$, as shown in Fig. 3. The real operating temperature can reach $80 \mathrm{~K}$.

Detectivity is the signal-to-noise ratio (SNR). A more useful figure of merit is the normalized detectivity $D^{*}$, which normalizes the detector area and band width.

$$
D^{*}=\frac{R_{\mathrm{p}} \sqrt{A_{\mathrm{d}} \Delta f}}{i_{\mathrm{n}}},
$$

where $R_{\mathrm{p}}$ is the responsivity of the detector, $A_{\mathrm{d}}$ is the area of the detector, $\Delta f$ is the bandwidth of the measurement, and $i_{\mathrm{n}}$ is the noise current which is supposed to be the shot noise and can be expressed as

$$
i_{\mathrm{n}}=\sqrt{4 e I_{\mathrm{d}} g_{\mathrm{n}} \Delta f},
$$

where $I_{\mathrm{d}}$ is the average dark current and $g_{\mathrm{n}}$ is the noise gain, which is proportional to the photoconductive gain $g$.

The advantage of $D^{*}$ as a figure of merit is that it is normalized to an active detector area of $1 \mathrm{~cm}^{2}$ and a noise

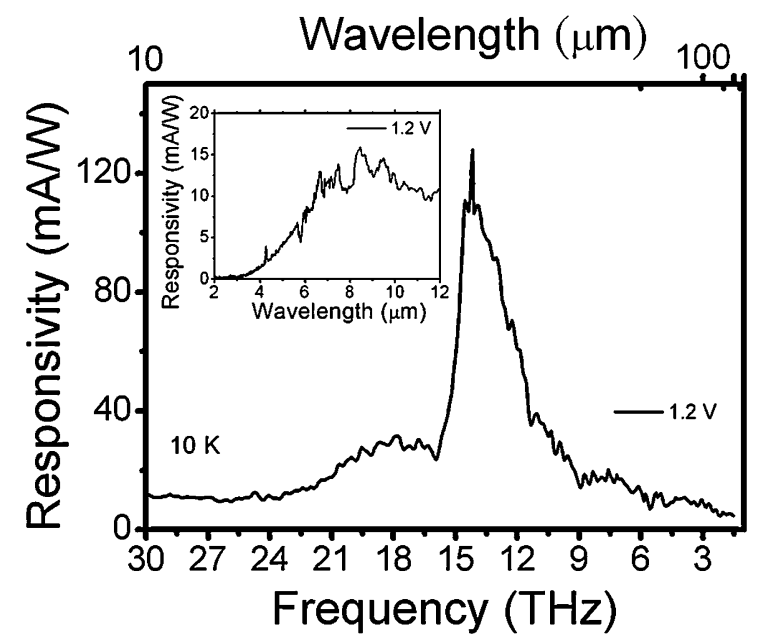

Fig. 4. Experimental responsivity spectra obtained under a bias of $1.2 \mathrm{~V}$ at $10 \mathrm{~K}$. The inset is the MIR responsivity.

bandwidth of $1 \mathrm{~Hz}$. The responsivity of the device was measured under a $45^{\circ}$-edge-coupling scheme with closedcycle cryogenics and Bruker IFS 66v/s Fourier transform infrared spectroscopy (FTIR). The detector was attached to the closed-cycle cryostat system with a copper adhesive tape. The mid-infrared (MIR) and FIR radiations were illuminated on the detector to excite photoelectrons. Current was then transferred to voltage using a SRS Model SR-570 current preamplifier, Finally, the voltage signal was transported back to FTIR. The spectral responsivity of the QR detector was measured from 100 to $3 \mathrm{THz}$ and a high detectivity of $2.3 \times 10^{11} \mathrm{~cm} \mathrm{~Hz}^{1 / 2} / \mathrm{W}$ was obtained at a peak wavelength of $21 \mu \mathrm{m}, 10 \mathrm{~K}$ and $1.2 \mathrm{~V}$ bias. The peak responsivity is observed at $127 \mathrm{~mA} / \mathrm{W}$, as shown in Fig. 4. Therefore, a prototype of terahertz photodetector is demonstrated by $\operatorname{In}(\mathrm{Ga}) A s$ QR with band gap tailorable property, this is a promising structure to replace the QD and the photoresponse of the detector can be extened to $\mathrm{THz}$ range. 
The normal incidence and broadband terahertz detector was fabricated using $\operatorname{In}(\mathrm{Ga})$ As quantum rings. The cutoff response can be operated up to $3 \mathrm{THz}(100 \mu \mathrm{m})$ at $10 \mathrm{~K}$. Moreover, the quantum ring $\mathrm{THz}$ detector has the potential for tailoring its cutoff frequency by adjusting the thin GaAs capped layer.

\section{Acknowledgement}

This work was supported by the National Science Council under Contract No. NSC-96-2120-M-002-003.

1) M. Herrmann, M. Tani, and K. Sakai: J. Appl. Phys. 91 (2002) 1247.

2) D. M. Mittleman, R. H. Jacobsen, and M. C. Nuss: IEEE J. Sel. Top. Quantum Electron. 2 (1996) 679.

3) P. H. Siegel: IEEE Trans. Microwave Theory Tech. 52 (2004) 2438.

4) D. L. Woolard, E. R. Brown, M. Pepper, and M. Kemp: Proc. IEEE 93 (2005) 1722

5) A. B. Weerasekara, M. B. M. Rinzan, S. G. Matsik, A. G. U. Perera, M. Buchanan, H. C. Liu, G. von Winckel, A. Stintz, and S. Krishna: Infrared Phys. Technol. 50 (2007) 194.

6) A. G. U. Perera, S. G. Matsik, B. Yaldiz, H. C. Liu, A. Shen, M. Gao,
Z. R. Wasilewski, and M. Buchana: Appl. Phys. Lett. 78 (2001) 2241.

7) S. G. Matsik, M. B. M. Rinzan, A. G. U. Perera, H. C. Liu, Z. R. Wasilewski, and M. Buchanan: Appl. Phys. Lett. 82 (2003) 139.

8) G. Airyawansa, A. G. Unil Perera, G. S. Raghavan, G. Von Winckel, A. Stintz, and S. Krishna: IEEE Photonics Technol. Lett. 17 (2005) 1064.

9) J. Philips, K. Kamath, and P. Bhattacharya: Appl. Phys. Lett. 72 (1998) 2020.

10) A. Lorke, R. Blossey, J. M. Garcia, M. Bichler, and G. Abstreiter: Mater. Sci. Eng. B 88 (2002) 225.

11) S. Viefers, P. Koskinen, P. Singha Deo, and M. Manninen: Physica E 21 (2004) 1.

12) R. J. Warburton, C. Schulhauser, D. Haft, C. Schaflein, K. Karrai, J. M. Garcia, W. Schoenfeld, and P. M. Petroff: Phys. Rev. B 65 (2002) 113303.

13) J. M. Llorens, C. Trallero-Giner, A. Garcia-Cristobal, and A. Cantarero: Phys. Rev. B 64 (2001) 035309.

14) D. Granados and J. M. Garcia: Appl. Phys. Lett. 82 (2003) 2401.

15) K. Takehana, F. Pulizzi, A. Patane, M. Henini, P. C. Main, L. Eaves, D. Granados, and J. M. Garcia: J. Cryst. Growth 251 (2003) 155.

16) D. Granados, J. M. Garcia, T. Ben, and S. I. Molina: Appl. Phys. Lett. 86 (2005) 071918.

17) R. Blossey and A. Lorke: Phys. Rev. E 65 (2002) 021603.

18) J. H. Dai, J. H. Lee, and S. C. Lee: IEEE Photonics Technol. Lett. 20 (2008) 165 